\section{Desigualdades sociais e cobertura vacinal: uso de inquéritos domiciliares}

\section{Social inequalities and vaccination coverage: utilization of household surveys}

\section{José Cassio de Moraes}

\section{Manoel Carlos Sampaio de Almeida Ribeiro}

Departamento de Medicina Social da Faculdade de Ciências Médicas da Santa Casa de Misericórdia de São Paulo

Correspondência: Rua Cesário Mota Jr 616 andar São Paulo Brasil. Email jcassiom@uol.com.br

\section{Resumo}

O Programa Nacional de Imunização, com mais de três décadas de existência, tem desempenhado papel fundamental no controle de importantes doenças imunopreveníveis, atingindo atualmente altas taxas de cobertura vacinal em praticamente todo território nacional. Esta situação instaura a necessidade de se aprimorar as estratégias de avaliação do programa, tanto na busca de indicadores mais precisos, quanto na mensuração e no entendimento das desigualdades sociais relacionadas à cobertura vacinal. Entende-se que a efetividade do programa de imunização, mensurada através da cobertura vacinal de uma população, está condicionada pelo sistema de saúde, pelo próprio programa de imunização e pelas características da população. Inquéritos domiciliares se colocam como uma das melhores estratégias para aprofundar o estudo das desigualdades. Contudo, a escolha da forma de mensurar a desigualdade dá-se em função dos objetivos do estudo. Assim, pode-se tanto avaliar como a vacinação se distribui na população sem distinguir grupos sociais, quanto estimar as desigualdades relativas e absolutas concernentes a grupos socioeconômicos. Por exemplo, os inquéritos de cobertura vacinal realizados em 1998 e 2002 no município de São Paulo, além de mensurarem um ligeiro aumento da cobertura vacinal no período, possibilitaram avaliar que as desigualdades em função de estratos socioeconômicos se modificaram sensivelmente. Em 1998 os estratos mais rico e mais pobre apresentavam as coberturas mais baixas. Em 2002, diferentemente, houve uma clara tendência de aumento da cobertura conforme a piora do estrato socioeconômico. 


\section{Introdução}

O uso de vacinas no Brasil remonta ao ano de 1804, quando as primeiras vacinações contra a varíola foram realizadas com a chegada do vírus vaccinio de Portugal, nos braços de escravos ${ }^{1}$.

Nestes mais de dois séculos várias centenas de milhões de doses de vacina foram aplicadas. Em 1968, o Estado de São Paulo instituiu o Programa Estadual de Imunização, definindo o elenco das vacinas a serem aplicadas e o calendário de imunização. Em 1973, o Ministério da Saúde estabeleceu o Programa Nacional de Imunização (PNI), com objetivo de promover o controle do sarampo, tuberculose, difteria, tétano, coqueluche e poliomielite, além da manutenção da erradicação da varíola² .

O programa "tem a característica de ser universal, sem distinção de qualquer natureza. As vacinas que compõem o atual calendário estão disponíveis em todos os postos de vacinação, e equipes móveis se deslocam para as áreas de difícil acesso quando necessário" ${ }^{2}$.

O controle de doenças transmissíveis tem nos programas de imunizações e de vigilância epidemiológica dois componentes fundamentais:

O conhecimento preciso da cobertura vacinal em menores de um ano e de um ano de idade é um dos elementos importantes para o programa de vigilância epidemiológica, na medida em que permite acompanhar o acúmulo de suscetíveis na população, bem como aquilatar até que ponto a imunidade de massa está se constituindo em barreira efetiva para a interrupção da transmissão das doenças preveníveis por imunização.

A cobertura vacinal é calculada, tradicionalmente, pelo numero de doses aplicadas, dividido pela população alvo. No caso das vacinas de múltiplas doses, o calculo é feito com o registro da terceira ou da segunda dose.

Rotineiramente, os dados de cobertura vacinal são obtidos a partir dos dados de produção dos serviços de cada uma das unida- des de vacinação e das estimativas populacionais do IBGE, ou então pelo número de recém-nascidos do SINASC. Essa informação é consolidada nas esferas municipal, estadual e federal, pelo aplicativo SI-API desenvolvido pelo Ministério da Saúde.

Os dados disponíveis para 2006 nos indicam, com algumas exceções, uma cobertura vacinal excelente em todas as unidades federativas, e com freqüência ultrapassa $100 \%^{3}$.

Contudo, a construção desse indicador apresenta vários problemas. Alguns são derivados da concepção do sistema de informação e outros do processo de coleta.

O Sistema de Informação utiliza para medir a cobertura vacinal o numero de doses aplicadas e não crianças vacinadas, tornando-se assim impossível saber, em termos coletivos, a situação vacinal das crianças e determinar, por exemplo, o percentual de crianças completamente vacinadas..

O numerador da fração pode estar alterado por erros no registro de doses aplicadas. Os erros ocorrem no tipo de imunobiológico aplicado à idade, e a dose. Em algumas situações especiais também podem acontecer erros na consolidação manual das doses aplicadas das unidades de vacinação de um município.

O denominador pode estar sub ou super dimensionado por erros na estimativa populacional da população alvo nos anos intercensitários. As estimativas populacionais para menores de um ano e de um a quatro anos estão sujeitas às variações de fecundidade, fluxo migratório etc. Os registros de nascimento em algumas partes do país são incompletos e não refletem a real taxa de natalidade.

Os resultados destes erros geram coberturas vacinais superestimadas, acima de $100 \%$, como, por exemplo, a cobertura com o BCG para o Brasil em 2006, ou coberturas super ou subestimadas nos Estados.

Outro aspecto relevante na analise da cobertura vacinal é a diversidade de condições de vida entre os municípios e internamente a um município, especialmente 
os de médio e grande porte. Essas diferentes situações podem gerar diferentes coberturas vacinais em um território municipal e que não são reveladas pela média. É possível que nas áreas com piores condições de vida o acesso ao programa de imunizações seja diferenciado em relação a outras áreas da cidade. Mesmo que os dados dos serviços próprios possam dar uma idéia dessas diferenças, estariam excluídas as informações de crianças atendidas em outras modalidades, tais como clínicas particulares de vacinação, convênios etc., que, apesar de ser uma exigência legal, não informam as autoridades municipais as vacinas aplicadas em suas instituições. Alem disso, as crianças que, embora residindo na área de influência do serviço, recebem suas vacinas em serviços localizados em outras áreas, visto não haver adscrição de clientela estabelecida.

A avaliação da cobertura vacinal por meio de inquéritos domiciliares realizados periodicamente permite estimar a real proporção de crianças vacinadas bem como medir as desigualdades sociais existentes na cobertura vacinal

\section{Desigualdades sociais em Saúde e cobertura vacinal}

As desigualdades sociais em saúde podem ser entendidas como aquelas diferenças produzidas pela inserção dos indivíduos na sociedade e que se relacionam com a distribuição de poder e propriedade ${ }^{4}$.

Dois enfoques têm sido habitualmente utilizados nos estudos das desigualdades: as desigualdades referentes ao estado de saúde e perfil patológico da população e as desigualdades no acesso aos serviços de saúde, que são diretamente influenciados pela política de saúde e pela organização dos serviços ${ }^{5}$.

Travassos e Martins declaram que, em linhas gerais, os determinantes da utilização de um serviço de saúde podem ser descritos em termos de: (a) necessidade de saúde - morbidade, gravidade e urgência; (b) usuários - características demográficas, geográficas, culturais, econômicas e psíquicas; (c) prestadores de serviços - características demográficas, tempo de graduação, especialidade, tipo de prática; (d) organização - recursos disponíveis, oferta, acesso geográfico e social; (e) política - tipo de sistema de saúde, financiamento, distribuição dos recursos ${ }^{6}$.

Neste sentido, entende-se que os fatores que interferem na cobertura vacinal podem ser agrupados em três tipos de condicionantes, do mais geral para o mais específico: sistema de saúde (política), o grau de estruturação das atividades relativas à vacinação (prestadores de serviços e organização) e os usuários.

A cobertura vacinal é na realidade um indicador de acesso ao Programa Nacional de Imunização, podendo ser indicativo da efetividade do "programa de imunização”. Incidência e mortalidade, por sua vez, podem ser consideradas indicadores das medidas de controle de doenças que incluem a assistência médica, as ações de vigilância epidemiológica e as próprias atividades de vacinação.

A vacinação é uma atividade, ou melhor, um serviço, de caráter preventivo. Assim, o usuário irá procurá-la na medida em que esta for uma necessidade de saúde. Ou seja, entende-se que cabe ao usuário, em primeira instância, a decisão de buscar a vacinação disponível nos serviços de saúde. Isto significa que a utilização de tal serviço é controlada pelo usuário, sendo que o grau de uso depende de suas características (incluindo o quanto vacinar é uma necessidade), e do "sistema de barreiras" existentes no acesso ao serviço ${ }^{6}$.

O contexto mais geral deste processo é dado pelo sistema de saúde. Por sistema de saúde entende-se tanto a forma quanto a estrutura utilizadas por cada país no cuidado da saúde da sua população, sendo particularmente importante o papel do Estado dentro do sistema.

A vacinação da população é historicamente, e em função do seu caráter coletivo uma intervenção de saúde pública? ${ }^{7}$. Desta forma, sistemas de saúde que pos- 
suem uma expressiva saúde pública conseguem produzir melhores indicadores de cobertura vacinal, como os casos da Inglaterra, Itália e Brasil ${ }^{2,8,9}$, dependendo, obviamente, das características do conjunto de atividades de vacinação e características populacionais.

Inserida no sistema de saúde, a vacinação se situa como uma das atividades de atenção primária, dependente, assim, do grau de estruturação deste nível de atenção no setor público e da sua oferta no setor privado. No contexto do sistema de saúde brasileiro, esta intervenção é guiada pelos princípios de universalidade e eqüidade.

Segundo Barata, pautando-se em Whitehead, a eqüidade implica o reconhecimento de que os indivíduos possuem diferentes necessidades e que, portanto, a partilha de recursos precisa considerar tais diferenças, não resolvidas mediante uma partilha igualitária ${ }^{4}$. Isto significa que todos têm direito à vacinação e que o Programa Nacional de Imunização deveria contemplar as diferentes necessidades criadas através de processos produtores de desigualdades sociais.

Na maioria dos sistemas de saúde as atividades relativas à vacinação são estruturadas na forma de um programa ${ }^{10}$. A existência de um programa nacional que dê conta das diferenças locais parece ser a forma mais associada a melhores coberturas vacinais. O quanto o programa está associado e integrado ao sistema de saúde, o quanto ele é centralizado e vertical varia bastante, sendo encontrados bons resultados de cobertura vacinal nas duas situações ${ }^{11}$. As campanhas, por exemplo, associadas em geral a programas mais centralizados e verticalizados, têm sido utilizadas para facilitar o acesso à vacinação ${ }^{8,12,13}$.

Estas características reforçam a idéia de que a vacinação, ainda que condicionada pelo sistema de saúde, possui, contudo, certa autonomia, conseguindo bons resultados mesmo em contextos desfavoráveis.

Outrossim, seria interessante investigar as especificidades desta intervenção dentro de distintos contextos políticos, econômicos e de sistemas de saúde. De início, cabe apenas conjeturar que esta autonomia se deve, por um lado, à característica essencialmente biomédica desta intervenção e, por outro, à grande legitimidade* que esta intervenção possui socialmente, devido, sobretudo, aos seus bens sucedidos resultados no controle de doenças.

Ao se considerar os usuários, a vacinação é entendida como uma intervenção que em si visa melhorar as condições de vida, ainda que por elas sejam condicionadas. Ter acesso a serviços de saúde e à vacinação significa, em si, uma melhor condição de vida.

Em geral, segmentos populacionais com níveis econômicos e socioculturais mais baixos estão associados a menores coberturas vacinais. No entanto, esta relação não é linear, cabendo ao sistema de saúde e ao "programa de vacinação" dar conta desta complexidade.

Segundo Andersen \& Newman, citados por Travassos, as condições e os estilos de vida dos usuários poderiam ser considerados os meios disponíveis através dos quais eles obtêm a vacinação ${ }^{6}$.

A relação entre uma condição econômica desfavorável e uma menor cobertura vacinal pode ser compreendida, sob a perspectiva da intervenção, como um aspecto da condição de vida que dificulta o acesso a ela. Isto é, piores condições econômicas podem significar uma menor oferta de serviços e/ou uma maior dificuldade de acessar à intervenção ${ }^{6}$.

Segundo Donabedian, acessibilidade é a possibilidade de se obter a intervenção quando necessária, de forma fácil e conveniente, no que tange tanto à oferta de serviços, como à dificuldade de acessá$\operatorname{los}^{14}$. Todavia, face às distinções anteriormente apresentadas, considera-se que os indicadores de condições de vida, embora avaliem características individuais e populacionais que interferem no acesso,

* De forma contra-hegemônica começam a surgir importantes questionamentos acerca do impacto desta intervenção, até do ponto de vista ecológico, e da sua própria legitimidade, aspectos que mereceriam ser também estudados ${ }^{10,11}$. 
excluem as características de oferta e organização dos serviços.

A relação entre os estilos de vida, concretizados em comportamentos, hábitos e atitudes, e a cobertura vacinal pode ser melhor sistematizada, também do ponto de vista da intervenção, através de dois conceitos, mais uma vez emprestados de Donabedian: aceitabilidade e legitimidade ${ }^{14}$.

A aceitabilidade está condicionada pela adaptação da intervenção aos desejos, expectativas e valores individuais e familiares e, assim, diz respeito a como cada indivíduo se submete à intervenção. Obviamente, a acessibilidade interfere no grau de aceitabilidade da intervenção ${ }^{14}$.

A legitimidade, por sua vez, refere-se ao modo pelo qual a sociedade e os grupos sociais vêem esta intervenção. Ela se vincula àquilo que é desejável para toda sociedade e, portanto, às formas pelas quais cada sociedade regula e media a relação indivíduo/sociedade ${ }^{14}$.

É claro que, num sentido mais amplo, a acessibilidade também está condicionada pela aceitabilidade e legitimidade da intervenção. Todavia, estes conceitos permitem diferenciar aspectos distintos das condições materiais de existência e, assim, compreender de que forma eles influenciam na acessibilidade à intervenção ${ }^{15}$.

Em síntese, entende-se que a efetividade do programa de imunização, mensurada através da cobertura vacinal de uma população, está condicionada pelo sistema de saúde, pelo próprio programa de imunização e pelas características da população. As diferenças identificadas de cobertura vacinal em função de variáveis individuais ou ecológicas expressam, na realidade, desigualdades no consumo deste serviço. A redução destas desigualdades entre grupos sociais coloca-se como um dos principais objetivos do PNI, podendo ser pensada particularmente por relação à posição socioeconômica, à raça, ao gênero e à localização geográfica ${ }^{16}$.

O estudo das coberturas vacinais segundo as condições de vida fornece uma oportunidade para a investigação acerca da concretização de políticas públicas compensatórias, entendidas como intervenções programadas e executadas preferencialmente pelo Estado, buscando diminuir as diferenças entre os grupos sociais produzidas por sua diferente inserção na organização social.

Idealmente, as políticas públicas de caráter coletivo deveriam ser capazes de compensar, na esfera do consumo de bens e serviços, as desigualdades geradas no processo de produção e reprodução social. Entretanto, uma série de condicionantes concretos relativos à existência e funcionamento dos serviços, possibilidades reais de utilização, comportamentos familiares e outros acabam por determinar um cumprimento apenas parcial dos objetivos visados por tais políticas. Assim sendo, é possível que, ao passar do plano da elaboração para o da execução, mesmo as intervenções que se pretendem universais tendam a se concretizar repetindo os mesmos padrões de desigualdade, embora de maneira atenuada.

\section{Como estudar as desigualdades}

Um primeiro aspecto consensual entre os autores é que a utilização de inquérito domiciliar viabiliza e potencializa a capacidade analítica de estudos de desigualda$\mathrm{de}^{5,16,18}$.

Tradicionalmente, recomenda-se a realização de inquérito de cobertura vacinal para responder questões fundamentais ao programa, tais como:

- Qual é a cobertura vacinal real e o número de susceptíveis para crianças aos 12 e aos 18 meses de idade?

- Qual é o acesso dessas crianças ao programa nacional de imunizações, e como se comporta a adesão ao mesmo até os 18 meses de idade?

- Qual é á diferença entre a cobertura vacinal estimada pelos inquéritos e os dados administrativos obtidos pelo sistema de informação do Programa Nacional de Imunização do Ministério da Saúde (SI-API)? 
Tabela 1 - Cobertura vacinal segundo imunobiológico e UF, Brasil; 2006.

Table 1 - Vaccination coverage according to immunobiological and State, Brazil; 2006

\begin{tabular}{|c|c|c|c|c|c|}
\hline Unidade Federação & BCG & $\begin{array}{l}\text { Contra } \\
\text { Hepatite B }\end{array}$ & $\begin{array}{l}\text { Oral Contra } \\
\text { Poliomielite }\end{array}$ & $\begin{array}{c}\text { Tetravalente } \\
\text { (DTP/Hib) }\end{array}$ & Tríplice Viral \\
\hline Rondônia & 101,13 & 95,49 & 102,19 & 100,71 & 103,47 \\
\hline Acre & 114,64 & 83,58 & 92,14 & 85,17 & 86,79 \\
\hline Amazonas & 112,27 & 76,53 & 76,1 & 81,44 & 92,48 \\
\hline Roraima & 113,53 & 82,65 & 88,61 & 87,45 & 91,94 \\
\hline Pará & 137,6 & 103,7 & 114,29 & 107,87 & 111,54 \\
\hline Amapá & 126,37 & 94,75 & 98,58 & 96,6 & 92,63 \\
\hline Tocantins & 90,03 & 88,38 & 91,46 & 91,56 & 84,06 \\
\hline Maranhão & 108,19 & 81,7 & 91,67 & 85,14 & 84,28 \\
\hline Piauí & 107,56 & 99,79 & 105,41 & 102,9 & 102,4 \\
\hline Ceará & 103,59 & 95,83 & 103,81 & 99,15 & 100,66 \\
\hline Rio Grande do Norte & 103,06 & 90,56 & 90,95 & 91,94 & 94,37 \\
\hline Paraíba & 109,28 & 97,86 & 101,86 & 100,59 & 102,85 \\
\hline Pernambuco & 113,9 & 99,4 & 107,07 & 105,55 & 107,52 \\
\hline Alagoas & 102,07 & 88,42 & 91,67 & 92,64 & 92,4 \\
\hline Sergipe & 106,95 & 95,03 & 100,08 & 99,69 & 96,51 \\
\hline Bahia & 104 & 91,54 & 96,82 & 95,61 & 101,09 \\
\hline Minas Gerais & 91,39 & 87,73 & 90,15 & 90,28 & 90 \\
\hline Espírito Santo & 100,38 & 96,57 & 109,83 & 100,33 & 102,89 \\
\hline Rio de Janeiro & 90,01 & 74,92 & 81,32 & 76,71 & 80,6 \\
\hline São Paulo & 99,67 & 93,03 & 94,4 & 94,64 & 97,29 \\
\hline Paraná & 93,85 & 89,65 & 92,14 & 92,38 & 89,88 \\
\hline Santa Catarina & 93,4 & 90,09 & 92,59 & 93,06 & 92,88 \\
\hline Rio Grande do Sul & 92,66 & 86,55 & 90,07 & 90,31 & 91,75 \\
\hline Mato Grosso do Sul & 99,04 & 96,74 & 99,32 & 99,41 & 98,92 \\
\hline Mato Grosso & 113,48 & 97,21 & 107,51 & 104,12 & 106,23 \\
\hline Goiás & 117,11 & 103,89 & 110,26 & 109,5 & 108,3 \\
\hline Distrito Federal & 108,71 & 94,31 & 98,79 & 94,08 & 98,21 \\
\hline Total & 102,59 & 91,09 & 95,7 & 94,19 & 96,03 \\
\hline
\end{tabular}

Fonte: Ministério da Saúde; 2007. Disponível em: http//www.saude.gov.br.

Além disso, pode-se investigar com inquérito os fatores que interferem na cobertura vacinal, associando-os às condições de vida e, assim, identificando e quantificando desigualdades sociais em saúde.

Há varias formas de mensurar as desigualdades sociais em saúde, que traduzem diferentes concepções teóricas e políticas sobre a desigualdade. Entretanto, não há consenso sobre uma definição única ou tida como correta $^{18}$.

As diferentes medidas podem ser agrupadas em duas grandes categorias: medidas de desigualdade em saúde senso estrito, e medidas de desigualdades socioeconômicas em saúde, as quais podem ser subdivididas em: medidas de associação, medidas de impacto potencial e medidas baseadas num ranking de variável socioeconômica ${ }^{16-18}$.

As medidas de desigualdade em saúde senso estrito, como o coeficiente de Gini, são medidas sintéticas das diferenças em saúde ao longo da população de indivíduos. Em geral, não consideram grupos sociais, sendo freqüentemente utilizadas por economistas. Podem ser interessantes para monitorar tendências e entre países onde os grupos sociais não sejam exatamente comparáveis, utilizando a informação de todos os indivíduos da população. Alguns autores defendem sua utilização em função de se poder prescindir da esco- 
lha de grupos a priori ${ }^{18}$.

As medidas de associação, por exemplo, razão e diferença de taxas e medidas de regressão, permitem a comparação do nível de saúde ou da freqüência do evento de interesse em grupos definidos através de variáveis socioeconômicas, utilizando medidas relativas e/ou absolutas. A principal limitação é não levar em consideração como os indivíduos se distribuem em cada categoria $^{18}$.

O Risco Atribuível Populacional tem sido tradicionalmente utilizado para avaliar o impacto de determinada diferença social na saúde da população ou num desfecho específico. Sua magnitude deriva de dois tipos de informação: da associação entre a variável socioeconômica e o desfecho de interesse e da distribuição dos indivíduos dentro de cada grupo socioeconômico. Assim, esta pode ser considerada uma medida que sintetiza a desigualdade, levando em consideração a distribuição e tamanho dos grupos socioeconômicos. No entanto, comparações entre diferentes populações, ou mesmo ao longo do tempo, demandam cuidado com a escolha do grupo de referência ${ }^{18}$.

Por sua vez, as medidas baseadas num ranking de variável socioeconômica, como a curva do índice de desigualdade e seu índice relativo e o índice de concentração, buscam sintetizar a distribuição do desfecho de interesse com função da variação de uma variável socioeconômica, sendo que está última possui uma ordenação dada por ranking hierárquico de categorias. Assume-se em geral uma linearidade da relação entre grupo social e o desfecho de saúde, ou seja, é preciso que os grupos sociais possam ser hierarquizados, o que é indiscutível com renda e educação, mas não em relação á etnia ou ao gênero ${ }^{18}$.

A escolha do tipo de medida de desigualdade se dá em função dos objetivos do estudo. Desta forma, se o objetivo for mensurar a desigualdade em saúde, poder-se-á utilizar medidas univariadas de desigualdade (primeira categoria). Se, por outro lado, o objetivo for estimar as desigualdades socioeconômicas em saúde, deveria ser privilegiado o uso dos outros três tipos de medidas, não havendo consenso sobre a primazia de alguma delas. Em linhas gerais, recomenda-se também que, para uma adequada monitorização das desigualdades e avaliação das intervenções, sejam utilizadas tanto medidas de diferenças absolutas, quanto relativas ${ }^{16,17}$.

\section{Cobertura Vacinal em São Paulo}

Os últimos dois inquéritos de cobertura vacinal, realizados em 1998 e 2002 no município de São Paulo, além de permitirem a obtenção de estimativas mais precisas, apontam para a complexidade das desigualdades atuais em relação cobertura vacinal $^{19,20}$.

Em geral, houve um ligeiro aumento da cobertura vacinal de 1998 para 2002. No entanto, as desigualdades em função de estratos socioeconômicos modificaram- se sensivelmente. Em 1998, o estrato mais rico e o estrato mais pobre apresentavam as coberturas mais baixas. Em 2002, diferentemente, há uma clara tendência de aumento da cobertura conforme a piora no estrato socioeconômico. Por exemplo, 68\% das crianças que vivem nas áreas de melhores condições de vida (estrato A) apresentavam esquema completo de vacinação aos 18 meses de idade, enquanto no estrato de piores condições de vida (estrato E) esse valor atingia $80 \%$ (Gráfico 1$)^{20}$.

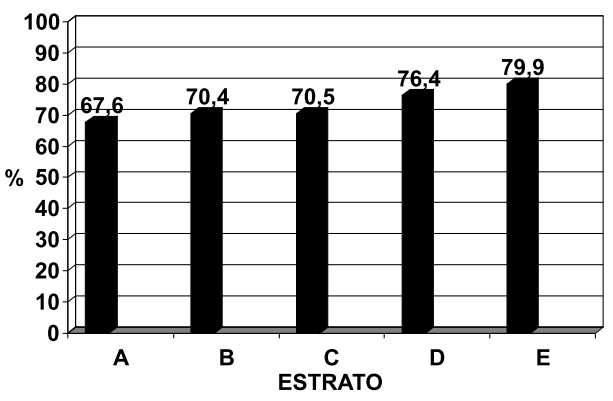

Gráfico 1 - Cobertura vacinal completa aos 18 meses de idade segundo estrato de condições de vida. Município de São Paulo, 2002

Chart1 - Vaccinationcoverage complete at 18 months of age accordingto lifeconditionsstratum. Municipality of São Paulo, 2002 
Os dois inquéritos no Município de São Paulo demonstraram reiteradamente coberturas baixas nas camadas com melhores condições de vida, contrariando o senso comum, o que por si representa um desafio na garantia do controle das doenças imunopreveníveis.

Ainda no sentido de expressar a complexidade das desigualdades na cobertura vacinal pode-se citar o comportamento da escolaridade da mãe nas proporções de cobertura em função do estrato. No estrato A, as crianças de mães alfabetizadas têm uma cobertura completa de $69 \%$ e as analfabetas de $62 \%$, diferença esta que não é observada nos demais estratos socioeconômicos (Gráfico 2$)^{20}$.

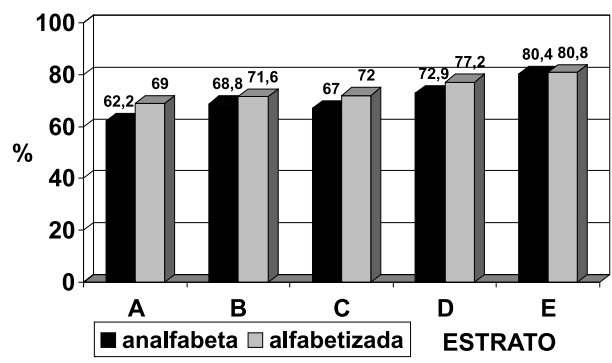

Gráfico 2 - Cobertura vacinal completa aos 18 meses de idade segundo escolaridade e estrato de condições de vida. Município de São Paulo, 2002

Chart 2 - Vaccination coverage complete at 18 months of age, according to schooling and life conditions stratum. Municipality of São Paulo, 2002.

Cabe desta forma olhar mais detalhadamente para a metodologia de dois inquéritos de cobertura vacinal que se propõem a avaliar a desigualdade no acesso à vacinação no município de São Paulo.

O primeiro foi realizado em 2002 e tinha por objetivo, além de estimar precisamente as coberturas vacinais para cada distrito de saúde (DS) do município de São Paulo, avaliar as desigualdades a partir estratos socioeconômicos definidos com base no DS de residência ${ }^{20}$.

Neste inquérito foi utilizado o processo de amostragem por conglomerados preconizados pela Organização Mundial de
Saúde para a realização de inquéritos de cobertura vacinal que pressupõe o sorteio de 7 crianças na faixa etária estabelecida para cada conglomerado num total de 30 conglomerados por área estudada (210 crianças $)^{20}$.

Os pressupostos utilizados para o cálculo do tamanho da amostra são: proporção de crianças vacinadas $=0,50$; nível de significância de 0,05 ; precisão da estimativa $=0,10$; e efeito do desenho $=2$; perdas $=10 \%$

Naquele momento, o município de São Paulo era dividido em 41 Distritos Sanitários (DS), e foi sorteada uma amostra para cada um deles.

Os estratos socioeconômicos foram construídos a partir de indicadores socioeconômicos relativos aos DS. Foram consideradas 14 variáveis pertencentes a seis domínios:

- Renda:

\% de chefes de família sem rendimentos $\%$ de chefes de família com renda inferior a 1,5 salários mínimos

- \% de chefes de família com renda acima de 20 salários mínimos

- Escolaridade:

- \% de chefes de família sem nenhum grau de escolaridade

- \% de chefes de família com menos de 4 anos de escolaridade (analfabetismo formal)

- \% de chefes de família com 15 anos ou mais de escolaridade

- Tipo de família:

- \% de famílias chefiadas por mulheres

- \% de famílias chefiadas por mulheres sem rendimentos

- Moradia:

- \% de domicílios com aglomeração (mais de 2 pessoas por cômodo)

- \% de domicílios subnormais (precários + improvisados + subnormais)

- Saneamento Básico:

- \% de domicílios sem coleta regular de lixo

- \% de domicílios sem abastecimento adequado de água

- \% de domicílios sem solução adequada 
para o esgotamento sanitário

- Composição populacional:

- \% de indivíduos com mais de 70 anos

Os dados correspondentes aos distritos administrativos foram agrupados e recalculados para os distritos de saúde (unidade amostral). Cada valor foi transformado para valores de $z$ (transformação normal), a fim de reduzir a variação decorrente das unidades de medida.

A análise de conglomerados ou "clusters" é uma técnica classificatória baseada no cálculo de vetores resultantes da disposição de $n$ elementos (neste caso, os distritos de saúde) no espaço de $p$ dimensões ( $p$ é o número de variáveis selecionadas para a análise), neste caso representado pelas variáveis socioeconômicas listadas acima.

Optou-se pelo método não hierárquico, no qual todas as variáveis são utilizadas simultaneamente para a classificação, chamado $k$-médias, pois os distritos são classificados a partir da distância do centróide do grupo. Nesse método, o centróide é estimado de tal maneira que as diferenças entre os valores individuais e o centróide são sempre inferiores às diferenças entre os centróides dos diversos conglomerados.

Nesse método é possível estabelecer a priori o número de conglomerados desejados, ou experimentar diferentes soluções até que a condição de parada, exposta anteriormente, seja atingida.

A execução da classificação foi feita utilizando-se o programa SPSS $®$ para Windows. Foram executadas soluções para três, quatro, cinco, seis e dez clusters, visto que para cada solução havia sempre um estrato com um único distrito de saúde incluído. Da comparação entre as soluções para quatro, cinco e seis clusters, e utilizando-se o coeficiente de variação do índice de exclusão entre os distritos em cada estrato, chegou-se à solução adotada.

A solução adotada distribui os distritos de saúde em cinco estratos, bastante diferentes em termos de condições de vida e cada um deles reunindo um número sufici- ente de crianças da amostra, garantindo assim poder estatístico às comparações feitas.

Além das informações sobre cada um dos imunobiológicos, foram coletadas informações sobre a escolaridade da mãe, a mãe trabalhar fora de casa, o número de pessoas por cômodo e a utilização de serviço privado.

Em função dos objetivos e do desenho, este inquérito permitiu estudar as desigualdades da seguinte forma:

- Avaliando as desigualdades de coberturas vacinais entre DS:

- Foram utilizadas regressões lineares e medidas de correlação entre as taxas de cobertura e os indicadores socioeconômicos dos DS. Por exemplo, encontrou-se um coeficiente de correlação de Spearman de $-0,43(\mathrm{p}=0,005)$ entre a proporção de chefes com renda maior que $20 \mathrm{sm}$ e a cobertura vacinal para esquema completo com um ano de ida$\mathrm{de}^{20}$.

- Avaliando as desigualdades de coberturas vacinais entre os estratos socioeconômicos de DS.

- Foram utilizadas medidas de associação (risco relativo e risco atribuível), estabelecendo-se como referência o grupo de melhor condição socioeconômica. Por exemplo, o estrato E apresentou uma cobertura vacinal para esquema completo com um ano de idade 1,25 maior que o estrato $\mathrm{A}^{20}$.

- Foram utilizadas análises estratificadas para especificar grupos definidos segundo atributos individuais dentro dos estratos socioeconômicos de DS. Por exemplo, quando se analisa a cobertura vacinal por estrato socioeconômico e trabalho fora da casa da mãe, observamos no estrato A uma diferença significativa. Para as mães que não trabalham as crianças apresentaram uma cobertura completa aos 18 meses de idade de $74 \%$, contra $62 \%$ para as que trabalham, ou seja, 1,19 vez maior. O mesmo não aconteceu nos demais estratos ${ }^{20}$.

- Avaliando as desigualdades de cober- 
turas vacinais em relação ao DS e ao conglomerado de residência e características socioeconômicas familiares. Foram utilizados modelos multinível de regressão logística para mensurar a importâncias das dimensões coletivas e individuais. Por exemplo, o modelo logístico final com três níveis, DS, conglomerado e indivíduo/família encontrou um pequeno efeito de conglomerado de 0,07 significa um aumento de 30\%* na chance de estar com esquema de vacinação completo para aqueles conglomerados que possuírem um efeito um desvio padrão acima da média. Em outras palavras, a existência do efeito sugere que a dimensão "conglomerado” pôde captar comportamentos familiares semelhantes em relação à cobertura vacinal para o esquema completo, independentes das variáveis individuais utilizadas ${ }^{21}$.

- Medidas de Impacto Populacional, como o Risco Atribuível Populacional, poderiam ter sido calculadas considerando tanto os estratos socioeconômicos de DS quanto os grupos definidos a partir de atributos individuais.

- Em relação às medidas sintéticas baseadas num ranking de variável socioeconômica, parece-nos que no caso da cobertura vacinal em São Paulo, onde há um claro gradiente favorável àqueles grupos com piores condições socioeconômicas, a utilização das mesmas pouco acrescentaria ao entendimento das desigualdades.

O inquérito a ser realizado em 2007 em todas as Capitais Federais pretende aprofundar a avaliação das desigualdades da cobertura vacinal relacionadas a grupos socioeconômicos, à raça e ao gênero. Para tanto, a estratégia de amostragem irá se basear em cinco estratos socioeconômicos construídos com base em indicadores dos setores censitários do IBGE, e que, funda- mentalmente, permitirão uma adequada representatividade na amostras destes estratos para cada um dos municípios.

A definição dos 5 estratos sociais será baseada na proporção de chefes de família com mais de 20 salários mínimos, na proporção de chefes de família com mais de 17 anos de estudo, e na média da renda nominal dos responsáveis pelos domicílios em cada um dos setores censitários. Estas variáveis são consideradas como discriminadoras das condições sociais. Serão atribuídos postos em ordem decrescente para cada uma das duas variáveis, e depois será efetuada a soma dos postos. Com essa soma, os setores censitários serão divididos em quintis. $\mathrm{O}$ primeiro quintil será o estrato $\mathrm{A}$ (menor pontuação e melhores condições de vida) e o último quintil o estrato $\mathrm{E}$ (maior pontuação e piores condições de vida).

O passo seguinte será construir conglomerados com pelo menos 56 crianças na faixa etária de 1 a 4 anos. Embora a metodologia consista em entrevistar 7 crianças da coorte de interesse em cada um dos 30 conglomerados, sabendo-se que passados alguns anos após o censo, parte das famílias pode haver mudado de endereço, parte das crianças pode ter morrido etc., opta-se por formar conglomerados compostos de um ou mais setores que contenham um número maior de crianças na idade alvo do estudo e residentes na área de interesse

A obtenção da amostra pressupõe nesta fase duas etapas: o sorteio dos conglomerados e o sorteio de um ponto inicial a partir do qual se dará início à busca das 7 crianças da coorte de interesse. Definidos os conglomerados (síntese de setores censitários com numero determinado de crianças), proceder-se-á ao sorteio sistemático dos mesmos utilizando-se no procedimento de amostragem a fração amostral correspondente (total da população $=30$ )

O IBGE também disponibiliza os mapas digitalizados dos setores sorteados a

* O efeito aleatório é mensurado em variância (0,07); portanto, através de sua raiz quadrada, obtém-se o desvio padrão 0,265. No modelo logístico multinível, a variação de um desvio padrão acima da média representa adicionar 0,265 aos coeficientes (betas). Através da exponencial do coeficiente obtém-se um odds ratio de 1,30, isto é, uma chance $30 \%$ maior ${ }^{21}$. 
fim de possibilitar a realização da segunda etapa do sorteio e a definição dos itinerários. Serão, então, numeradas todas as quadras e as faces de cada uma delas. A segunda etapa consiste em sortear uma quadra em cada conglomerado e um lado nessa quadra para dar início ao itinerário de busca das crianças pertencentes à coorte de interesse.

Serão também coletadas informações sobre a escolaridade da mãe ou do responsável pela criança, um inventário de bens moveis da família e aglomeração intradomiciliar, permitindo uma classificação da situação socioeconômica da família da criança.

As desigualdades de cobertura vacinal relativas à cor/raça poderão ser inves- tigadas a partir da referência da cor das crianças por parte da mãe ou responsável.

Por sua vez, as desigualdades de gênero poderão ser investigadas a partir do sexo da criança e através da compreensão da importância de homens e mulheres na estrutura de cuidado da criança, detalhada pelo número de filhos, estado conjugal, mulher trabalhando fora e presença da avó no domicílio.

O desafio que ora se coloca para pesquisadores e para os profissionais de saúde é o de refletir sobre a melhor forma de mensurarmos e avaliarmos as desigualdades no acesso ao PNI no município, a partir do aprofundamento permitido e potencializado pela utilização de inquéritos populacionais

\section{Referências}

1. Temporão JG, Programa Nacional de Imunização (PNI): origens e desenvolvimento. História, Ciência e Saúde 2003;1(2):601-17.

2. Brasil. Programa Nacional de Imunização - 30 anos. Secretaria de Vigilância a Saúde. Ministério da Saúde; 2003.

3. Brasil. Ministério da Saúde; 2006. Disponível em: http// www.datasus.gov.br.

4. Barata RB. Iniqüidade e saúde: A determinação social do processo saúde doença. Palestra; 2001.

5. Barata, RB. Desigualdades Sociais em Saúde. In: Campos, GWS et al.(org.). Tratado de Saúde Coletiva. São Paulo: Hucitec; Rio de Janeiro: Ed. Fiocruz; 2006.

6. Travassos C, Martins M. Uma revisão sobre os conceitos de acesso e utilização de serviços de saúde. Cad Saude Pública 2004; 20(2): S190-8.

7. Plotkin SA, Orestein W A. A short history of vaccination. Vaccines: NB Saunders Company; 2004.

8. Pebley AR, Goldman N, Rodríguez G. Prenatal and delivery care and childhood immunization in Guatemala: Do family and community matter? Demography 1996; 33(2): 231-47.

9. Salmaso S. Infant immunization coverage in Italy: estimates by simultaneous EPI cluster surveys of regions. ICONA Study Group. Bull World Health Organ 1999; 77(10): 843-51.
10. Streefland PH, Chowdhury AMR, Ramos-Jimenez P. Patterns of vaccination acceptance. Soc Sci Med 1999; 49: 1705-16.

11. Streefland PH. Public doubts about vaccination safety and resistance against vaccination. Health Police 2001; 55: 159-72.

12. McQuestion M, Jones RE. A Dynamic, Multi-level Analysis of Recent Immunization Trends in Colombia. Soc Biol 1998;45(1-2): 39-59.

13. Moraes JC, Barata RC, Ribeiro MCSA, Castro PC. Cobertura Vacinal no primeiro ano de vida em quatro cidades do estado de São Paulo, Brasil. Rev Panam Salud Rev Panam Salud Publica/Pan Am J Public Health 8(5), 2000: 332-341.

14. Donabedian A. The seven pillars of quality. Arch Pathol Lab Med 1990; 114: 1115-8.

15. Donabedian A.The quality of care. How can it be assessed? JAMA 1988; 260(12): 1743-48.

16. Regidor, E. Measures of Health Inequalities: part1. J Epidemiol Community Health 2004; 58: 858-61.

17. Regidor, E. Measures of Health Inequalities: part2. $J$ Epidemiol Community Health 2004; 58:900-03.

18. Schneider, MC; Salgado-Castilho, C; Bacallao, J; Loyola, E; Mujica, O; Vidaurre, M; Roca, A. Métodos de Medición de las Desigualdades de Salud. Rev Panam Salud Publica 2002; 12(6): 398-415 
19. Moraes JC, Barata RC, Ribeiro MCSA, Castro PC. Cobertura Vacinal no primeiro ano de vida em quatro cidades do estado de São Paulo, Brasil. Rev Panam Salud Pública 2000; 8(5): 332-41.

20. Barata RC, Moraes JC, Ribeiro MCSA, Simões O, Castro PC, Mendes JDV, Guibu IA, Gonçalves MJPR. Inquérito de cobertura vacinal no município de São Paulo, 2002. São Paulo: CEALAG; 2002.
21. Ribeiro, MCSA. Análise multinível da cobertura vacinal no município de São Paulo. [Tese de doutorado]. São

Paulo: Departamento de Medicina Preventiva da Faculdade de Medicina da USP; 2005. 